\title{
Gene expression profile of lipopolysaccharide-induced apoptosis of nucleus pulposus cells reversed by syringic acid
}

\author{
HENG ZHANG $^{1 *}$, HONG QIN $^{2 *}$, CHENGEN ZHOU $^{1}$, QIANG FENG ${ }^{1}$, \\ YUAN YANG $^{1}$, JINHUI SUI ${ }^{1}$ and YUCAI TANG ${ }^{1}$ \\ ${ }^{1}$ Department of Rehabilitation Medicine, Langdong Hospital of Guangxi Medical University, Nanning, \\ Guangxi Zhuang Autonomous Region 530028; ${ }^{2}$ Department of Health Care, Nanning Maternal and Child Health Hospital, \\ Nanning, Guangxi Zhuang Autonomous Region 530011, P.R. China
}

Received January 13, 2020; Accepted July 3, 2020

DOI: $10.3892 / \mathrm{mmr} .2020 .11632$

\begin{abstract}
Apoptosis of nucleus pulposus (NP) cells has an important role in the process of intervertebral disc degeneration (IDD), and the search for novel compounds to prevent apoptosis from occurring is urgently required. In the present study, syringic acid (SyrA) was found to exhibit no cytotoxicity on NP cells, and was able to reverse the cytotoxicity, as well as the abnormal expression of Bcl-2 and caspase-3, that were induced by lipopolysaccharide (LPS). The transcriptomes of each group were then analyzed using RNA-Seq. A total of 65 differentially expressed genes (DEGs) were identified in LPS-stimulated groups (LPS group vs. control group), 819 DEGs were identified in the SyrA-reversed groups (SyrA plus LPS group vs. LPS group), and a further 25 DEGs were identified in the SyrA plus LPS group compared with the control group. Reverse transcription-quantitative PCR validation indicated that the alterations in expression of uroplakin 3B-like 1 (UPK3BL1), voltage-dependent calcium channel subunit $\alpha-2 / \delta-1$ (CACNA2D1) and polo-like kinase 4 (PLK4) were consistent with the corresponding results of RNA-Seq, and that these genes were involved in both LPS-stimulation and SyrA-reversion processes. Kyoto Encyclopedia of Genes and Genomes analyses indicated that the DEGs in SyrA-reversed groups were involved in, amongst other pathways, 'Autophagy-other' and 'Apoptosis-multiple species'. In conclusion, the addition of SyrA to the NP cells co-incubated with LPS appeared to help prevent the abnormal expression
\end{abstract}

Correspondence to: Dr Chengen Zhou or Dr Qiang Feng, Department of Rehabilitation Medicine, Langdong Hospital of Guangxi Medical University, 60 Jinhu Road, Nanning, Guangxi Zhuang Autonomous Region 530028, P.R. China

E-mail: 243369941@qq.com

E-mail: 334754234@qq.com

*Contributed equally

Key words: syringic acid, apoptosis, nucleus pulposus cells, RNA-Seq of mRNAs and apoptosis that had been identified in NP cells incubated with LPS alone. The potential mechanism underlying the reversion of SyrA might be attributed to the regulation of CACNA2D1 and PLK4.

\section{Introduction}

Lower back pain is a common health problem worldwide. Chronic pain makes life difficult, and in some cases, it can even develop into disability: Statistics have shown that lower back pain has been one of the three leading causes of disability in the past two decades (1), directly leading to long-term heavy burdens for both the immediate family and in society at large. Intervertebral disc degeneration (IDD) is generally regarded as the predominant causative factor for lower back pain (2). The process of IDD is spontaneous with age, and both lack of vascularization and limited cell presence in the disc make self-repair impossible (3). When degeneration occurs, the homeostatic environment is disturbed and the balance between anabolism and catabolism is disrupted, leading to an increased apoptosis of nucleus pulposus (NP) cells accompanied by the loss of the extracellular matrix (ECM) $(3,4)$. NP cells are the major cells in NP tissue and have an important role in resisting mechanical loading by synthesizing ECM (4). The apoptosis of NP cells is considered crucial in the degeneration process (5). Thus, the search for new molecules able to prevent apoptosis is fundamental in the search to provide better therapeutic interventions against IDD.

Syringic acid (SyrA), a natural phenolic compound, exists in various plant species and possesses many biologically important properties. A recent review by Srinivasulu et al (6) details how SyrA has been shown to fulfill various roles as an anti-oxidant, anti-inflammatory, chemo-protective and anti-microbial compound. The chemo-protective activity of SyrA is effective on multiple cells with different functions. For example, pre-treatment with SyrA has been shown to reverse neuronal injury as well as the apoptosis of hippocampal neuronal cells, processes that were induced by oxygen-glucose deprivation/reperfusion, and the neuroprotective effect of SyrA was shown to be dose-dependent (7). In addition, $\mathrm{H}_{2} \mathrm{O}_{2}$-induced apoptosis of retinal ganglion cells, apoptosis of cardiomyocytes following hypoxia/reoxygenation treatment 
and apoptosis in rat renal ischemia-reperfusion injury have all been shown to be inhibited by pre-treatment or treatment with SyrA (8-10). However, to date, the effect of SyrA on NP cells and their apoptosis is unknown, although these aforementioned studies could suggest that this molecule might reverse the apoptosis of NP cells.

The present study investigated the role of SyrA in the lipopolysaccharide (LPS)-induced apoptosis of NP cells, and following SyrA treatment, the differentially expressed genes (DEGs) were analyzed using RNA-Seq analysis. Reverse transcription-quantitative PCR (RT-qPCR) and bioinformatics analyses were also performed to study the potential mechanisms involved. The results of the present study may provide fresh avenues for further exploring the reversion of apoptotic NP cells and the therapeutic intervention of IDD.

\section{Materials and methods}

Cells and cell culture. Human NP cells which had previously been isolated from NP tissues of patients with lumbar fracture (AO classification, B1) were kindly provided by Dr. Guo (Shanghai Changzheng Hospital, Shanghai, China) (11). NP cells were cultured in DMEM/F12 medium (Gibco; Thermo Fisher Scientific, Inc.) supplemented with $10 \%$ fetal bovine serum (Gibco; Thermo Fisher Scientific, Inc.) and 1\% penicillin-streptomycin (Gibco; Thermo Fisher Scientific, Inc.) at $37^{\circ} \mathrm{C}$ in an incubator in an atmosphere of $5 \% \mathrm{CO}_{2}$.

Cell viability. Cell Counting kit-8 (CCK-8; Beyotime Institute of Biotechnology) was used to evaluate cell survival. Briefly, NP cells were seeded in a 96 -well plate $\left(5 \times 10^{3}\right.$ cells/well $)$ and incubated overnight. Subsequently, LPS (Sigma-Aldrich; Merck KGaA) or SyrA (Sigma-Aldrich; Merck KGaA) (Fig. 2A) were added at a final concentration of $0,0.001$, $0.01,0.1,1,10$ and $100 \mu \mathrm{g} / \mathrm{ml}$ for LPS $(12,13)$, and 0,200 , 400 and $600 \mu \mathrm{M} / \mathrm{ml}$ for SyrA. After 3 days, $10 \mu \mathrm{l} \mathrm{CCK}-8$ reagent was added to each well and cultured for a further $2 \mathrm{~h}$. Subsequently, the plate was transferred into a microplate reader (BioTek Instruments, Inc.), and the absorbance at $450 \mathrm{~nm}$ was detected.

Western blot analysis. NP cells were seeded in a 6-well plate $\left(4 \times 10^{5}\right.$ cells/well) and treated with LPS $(0.01 \mu \mathrm{g} / \mathrm{ml})$, or with combined SyrA $(400 \mu \mathrm{M})$ and LPS $(0.01 \mu \mathrm{g} / \mathrm{ml})$ for $48 \mathrm{~h}$. Cell lysates were then prepared using radioimmunoprecipitation assay (RIPA) buffer (Sigma-Aldrich; Merck KGaA) and total protein concentration was determined using a BCA protein assay kit (Biosharp Life Sciences). A total of $10 \mu \mathrm{g}$ protein was separated by SDS-PAGE (12\% gels) and transferred to polyvinylidene fluoride membranes. Subsequently, these membranes were blocked in blocking buffer (Beyotime Institute of Biotechnology) at room temperature for $60 \mathrm{~min}$ and separately incubated overnight at $4^{\circ} \mathrm{C}$ with antibodies against caspase-3 (cat. no. 9662; 1:1,000), Bcl-2 (cat. no. 15071; 1:1,000), and GAPDH (cat. no. 5174; 1:1,000). The next day, the membranes were incubated with horseradish peroxidase-conjugated secondary antibody (cat. no. 7076; 1:1,000) at room temperature for $2 \mathrm{~h}$. All the antibodies were purchased from Cell Signaling Technology, Inc., and protein expression was normalized against GAPDH. Band densitometry was conducted and semi-quantified using ImageJ software version 1.8.0 (National Institutes of Health), and the relative gray value was used to represent the relative protein expression.

RNA-Seq analysis. NP cells were treated with LPS $(0.01 \mu \mathrm{g} / \mathrm{ml})$ or combined LPS $(0.01 \mu \mathrm{g} / \mathrm{ml}) / \operatorname{SyrA}(400 \mu \mathrm{M})$ for $48 \mathrm{~h}$; control cells were given no treatment. Total RNA from the three groups was isolated using an RNeasy Mini kit (Qiagen $\mathrm{GmbH}$ ) and poly A-containing mRNA molecules were purified using poly $\mathrm{T}$ oligo-attached magnetic beads. Next, the mRNA was fragmented into small pieces, and these fragments were reverse-transcribed into first-strand cDNA using RNA Seq First Strand Master Mix (Agilent Technologies, Inc.) following manufacturer's instructions. Subsequently, second-strand cDNA was synthesized using DNA polymerase I and RNase H. After an end-repair process, consisting of the addition of a single adenosine base, the products were purified and enriched with PCR to create the final cDNA library. Purified libraries were quantified using a Qubit ${ }^{\circledR}$ 2.0 Fluorometer (Thermo Fisher Scientific, Inc.) and validated using an Agilent 2100 Bioanalyzer (Agilent Technologies, Inc.) to confirm the size of the insert and calculate the molar concentration. Clusters were generated using the Illumina software cBot, with the library diluted to $10 \mathrm{pM}$ and then sequenced on an Illumina NovaSeq 6000 instrument (Illumina, Inc.). All gene level files were imported into the Agilent GeneSpring GX software version 11.5 (Agilent Technologies, Inc.) for further analysis. Only genes that fulfilled the two criteria $\left[\log _{2}\right.$ fold change (FC) (abs, absolute value) $\geq 1$ and $\mathrm{P}<0.05]$, were filtered as DEGs.

$R T-q P C R$. Total RNA from NP cells of each group was obtained using the RNAsimple Total RNA Extraction kit (Tiangen Biotech Co., Ltd.). Subsequently, $1 \mu \mathrm{g}$ total RNA was used to synthesize complementary DNA (cDNA) with a RT PCR kit (Applied Biosystems; Thermo Fisher Scientific, Inc.). Subsequently, $0.5 \mu \mathrm{l}$ of the resulting cDNA was added to a reaction system according to the instructions of the SYBR Premix Ex Taq ${ }^{\mathrm{TM}}$ II kit (Takara Biotechnology Co., Ltd.). Thermocycling conditions were as follows: $95^{\circ} \mathrm{C}$ for $60 \mathrm{sec}$, followed by 40 cycles at $95^{\circ} \mathrm{C}$ for $15 \mathrm{sec}$ and $60^{\circ} \mathrm{C}$ for $60 \mathrm{sec}$. Primers with the sequences listed in Table I were provided by Sangon Biotech Co., Ltd. Gene expression data were processed using the $2^{-\Delta \Delta \mathrm{Cq}}$ method (14) and normalized to GAPDH.

Bioinformatics analyses. Gene Ontology (GO) (15) and Kyoto Encyclopedia of Genes and Genomes (KEGG) (16) signaling pathway analyses were performed to analyze the biological functions and the potential relevant signaling pathways of the DEGs using clusterProfiler, a R/Bioconductor implementation. $\mathrm{P}<0.05$ was used as a threshold to screen relevant $\mathrm{GO}$ terms and KEGG pathways, and the top 30 pathways were selected for drawing.

Statistical analyses. Statistical analyses were performed using SPSS 19.0 software (IBM Corp.). The results are presented as the mean \pm standard deviation (SD). For comparisons between groups, one-way analysis of variance was used, followed by Tukey's multiple comparison tests. All the experiments, except 
Table I.Primer sequences for reverse transcription-quantitative PCR.

\begin{tabular}{ll}
\hline Primer name & \multicolumn{1}{c}{ Primer sequence (5'-3') } \\
\hline UPK3BL1 & F: TTCAGCAGCCACAACATCTC \\
UPK3BL1 & R: GCAGCCAATTTTGTTGGTT \\
CACNA2D1 & F: ACGCAGCAGTCCATATTCCTA \\
CACNA2D1 & R: GCCACAATAATGAAGGGTCTTCC \\
PPP1R9A & F: ATCGAACTGAGTTTCAGGCAC \\
PPP1R9A & R: GGTTCCATACCCATCTGCATAAA \\
WRN & F: CACAGCAGCGGAAATGTCCT \\
WRN & R: GAGCAATCACTAGCATCGTAACT \\
PLK4 & F: AAGCTCGACACTTCATGCACC \\
PLK4 & R: GCATTTTCAGTTGAGTTGCCAG \\
SLC9A6 & F: TCACCCTCACCATTCTCACA \\
SLC9A6 & R: CACTTCACAGCTCAGGGTCA \\
NR3C1 & F: ACAGCATCCCTTTCTCAACAG \\
NR3C1 & R: AGATCCTTGGCACCTATTCCAAT \\
GAPDH & F: GGAGCGAGATCCCTCCAAAAT \\
GAPDH & R: GGCTGTTGTCATACTTCTCATGG
\end{tabular}

F, forward; R, reverse.

RNA-Seq, were performed at least three times. $\mathrm{P}<0.05$ was considered to indicate a statistically significant difference.

\section{Results}

LPS induces apoptosis of NP cells. To confirm the appropriate concentration of LPS, NP cells were treated with $0,0.001,0.01$, $0.1,1,10$ and $100 \mu \mathrm{g} / \mathrm{ml}$ LPS. As shown in Fig. 1A, significant cell inhibition of LPS was observed at 0.01, 0.1 and 1, and $0.01 \mu \mathrm{g} / \mathrm{ml}$ LPS showed the greatest effect on the primary NP cells' viability. Hence, the $0.01 \mu \mathrm{g} / \mathrm{ml}$ concentration was selected for further studies. Western blot analysis showed that the protein level of caspase-3 significantly increased and that Bcl-2 expression levels significantly decreased (Fig. 1B), which suggested that there was an onset of apoptosis after LPS stimulation.

SyrA reverses LPS-induced apoptosis in NP cells. Before studying how SyrA (structure shown in Fig. 2A) affected LPS-induced apoptosis, its cytotoxicity on NP cells was evaluated. The results of the CCK- 8 assay indicated that there were no adverse cytotoxic effects on NP cells at any of the three concentrations; however, there was a significant proliferative effect (Fig. 2B). Subsequently, the effects of the three aforementioned concentrations of SyrA on LPS-induced NP cell apoptosis were studied; the highest rate of reversion of cell apoptosis was induced by $400 \mu \mathrm{M} \mathrm{SyrA} \mathrm{(Fig.} \mathrm{2C).} \mathrm{The} \mathrm{protein}$ levels of caspase-3 and Bcl-2 were subsequently detected, and the results indicated that their expression in NP cells was reversed following treatment with $400 \mu \mathrm{M}$ SyrA (Fig. 2D).

RNA-Seq analysis of NP cells treated with LPS. To develop a better understanding of the apoptosis reversal induced by
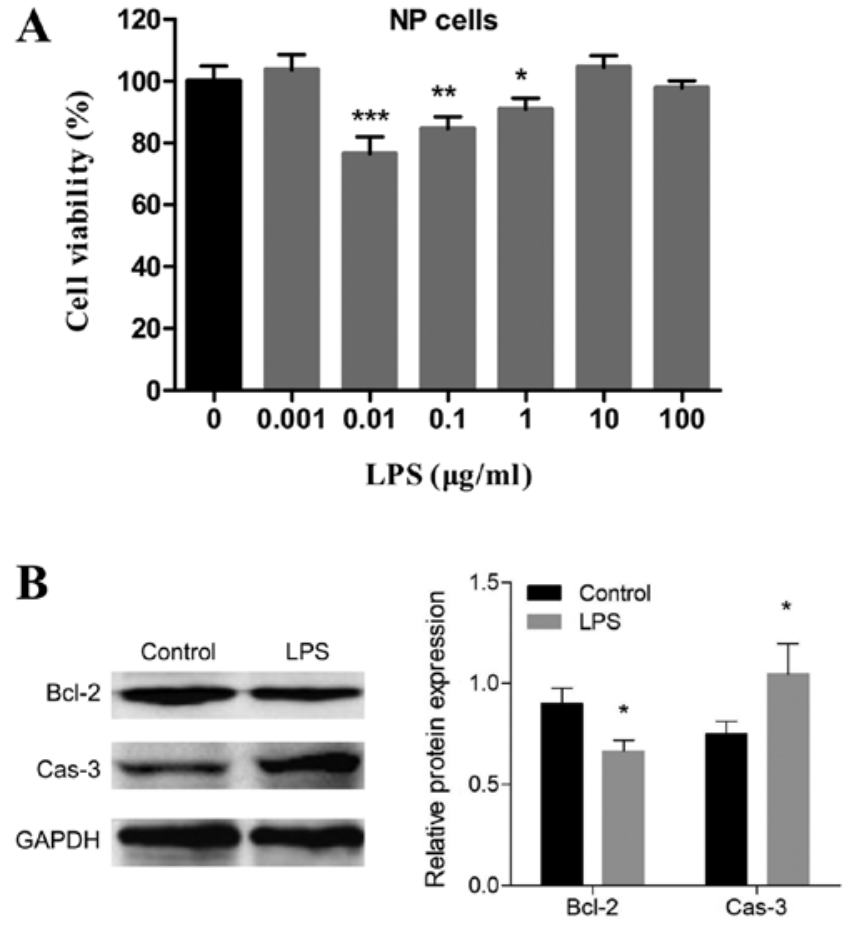

Figure 1. LPS induces the apoptosis of NP cells. (A) The effect of different concentrations of LPS on the viability of NP cells. (B) The effect of $0.01 \mu \mathrm{g} / \mathrm{ml}$ LPS on the protein expression of Bcl-2 and caspase- 3 genes in NP cells. ${ }^{*} \mathrm{P}<0.05,{ }^{* *} \mathrm{P}<0.01,{ }^{* * *} \mathrm{P}<0.001$. LPS, lipopolysaccharide; NP, nucleus pulposus.

SyrA, RNA-Seq of NP cells from LPS groups (LPS group vs. control group) was performed. As shown in Fig. 3A, 65 DEGs were screened, and the majority of them were downregulated following LPS treatment. Volcano plots and scatter plots (Fig. 3B and C) indicated that there were 20 upregulated (red dots) and 45 downregulated (blue dots) DEGs. Among the DEGs, five were previously unknown genes (data not shown), and the top 15 known DEGs are listed in Table II. The $\log _{2} \mathrm{FC}$ (abs) of the top 15 DEGs varied from 2.4 to 5.2, and most of the top 15 DEGs were downregulated.

RNA-Seq analysis of LPS-stimulated NP cells treated with SyrA. The effect of SyrA on LPS-stimulated NP cells was much more comprehensive compared with that of LPS on NP cells. A total of 819 DEGs from SyrA-reversed groups (SyrA plus LPS group vs. LPS group) were screened, and these are presented in the heat-map (Fig. 4A). Among the DEGs, 727 were upregulated (red dots) and 92 were down-regulated (blue dots); the resulting volcano plots and scatter plots (Fig. 4B and C) showed that the $\log _{2} \mathrm{FC}$ (abs) of the DEGs was 2-5. The unknown DEGs (named with alphanameric code, lacking a specific gene name) in the two groups reached 57 DEGs $(6.96 \%$; data not shown), and the top 15 known DEGs are presented in Table III. Notably, the upregulation of uroplakin 3B-like 1 (UPK3BL1) in LPS-stimulated NP cells $\left(\log _{2} \mathrm{FC}=5.136\right)$ was reversed by SyrA, resulting in its downregulation $\left(\log _{2} \mathrm{FC}=-5.558\right)$ ( Tabl es II and III). A similar trend could also be observed in the expression of the gene for voltage-dependent calcium channel subunit $\alpha-2 / \delta-1$ (CACNA2D1), with the $\log _{2} \mathrm{FC}$ changing from -4.206 to 3.468 (Tables II and III). These changes suggested that there might be a broad reversion of SyrA on LPS-resulted DEGs in NP cells. Thus, DEGs between the SyrA plus LPS 
A<smiles>COc1cc(C(=O)O)cc(OC)c1O</smiles>

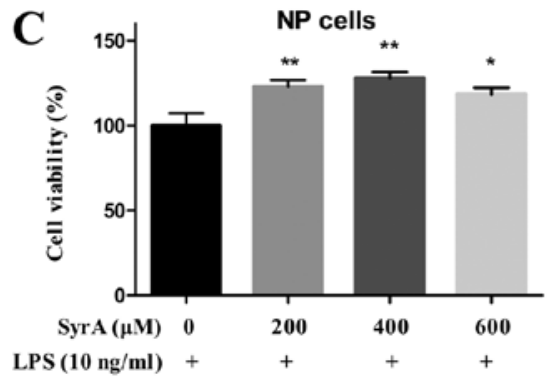

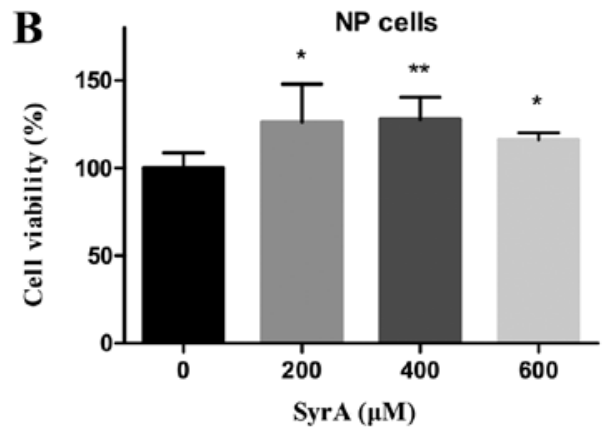

D

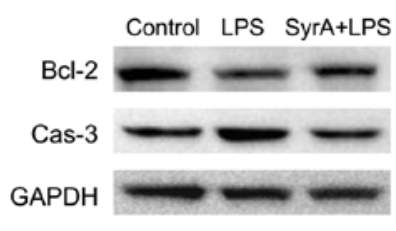

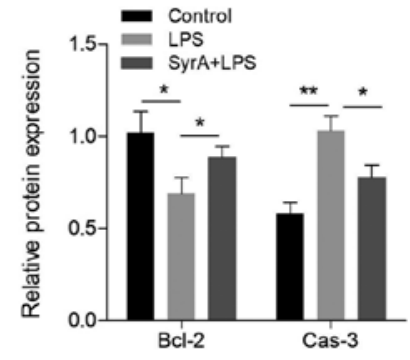

Figure 2. SyrA reversed the LPS-induced cytotoxicity and apoptosis of NP cells. (A) Molecular structure of SyrA. (B) Cytotoxicity of different concentrations of SyrA on NP cells. (C) The cytotoxicity on NP cells of $0.01 \mu \mathrm{g} / \mathrm{ml}$ LPS was reversed by 200, 400 or $600 \mu \mathrm{M}$ SyrA. (D) The protein expression of Bcl-2 and caspase-3 in LPS-simulated NP cells was reversed by $400 \mu \mathrm{M}$ syringic acid. ${ }^{*} \mathrm{P}<0.05,{ }^{* *} \mathrm{P}<0.01$. SyrA, syringic acid; LPS, lipopolysaccharide; NP, nucleus pulposus.

A

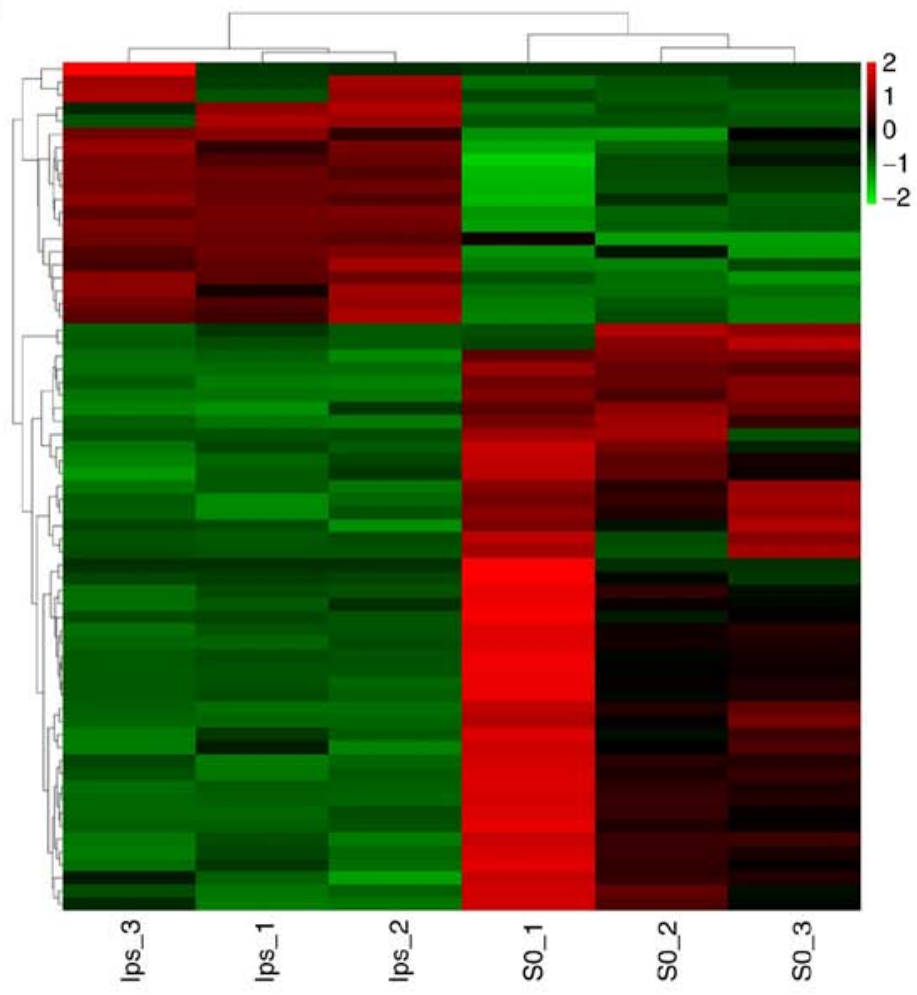

B Ips Vs so

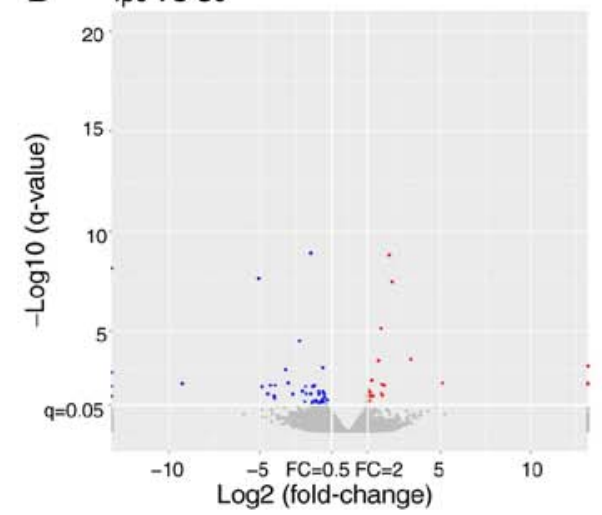

C Ips VS SO 20 $r=0.958$
$p<2.2 e-16$

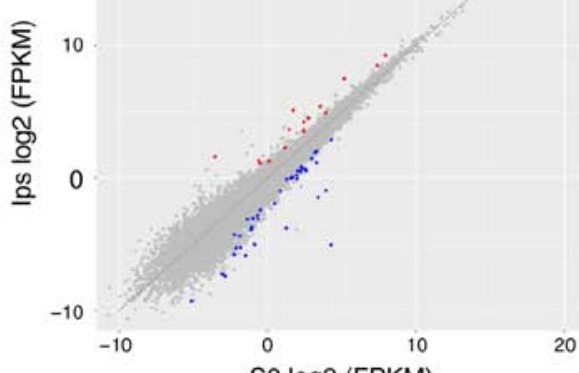

So $\log 2$ (FPKM)

Figure 3. RNA-Seq analysis of NP cells treated with LPS. (A) Heat map of DEGs from LPS-stimulated groups (LPS group vs. control group). (B) Volcano plots of 20 upregulated and 45 downregulated DEGs. The vertical lines represent abs $\left(\log _{2} \mathrm{FC}\right)=1$, and the thick horizontal line indicates $\mathrm{P}=0.05$. (C) $\mathrm{Scatter}$ plots of the genes with $\log _{2} \mathrm{FC}(\mathrm{abs})>1$ in LPS-stimulated groups. 'lps' indicates the LPS treatment group and 'S0' indicates the control group. NP, nucleus pulposus; LPS, lipopolysaccharide; DEGs, differentially expressed genes; abs, absolute; FC, fold change.

group and the control group were also analyzed. As shown in Fig. 5A and B, the total DEGs (red and blue dots) number was only 25, which indicated that SyrA could reverse most of the 65 DEGs resulting from LPS stimulation. 
Table II. Top 15 differentially expressed genes from lipopolysaccharide-stimulated groups.

\begin{tabular}{rllrrrr}
\hline No. & \multicolumn{1}{c}{ Gene ID } & Gene name & $\log 2$ FC & FC & P-value & Up/downregulated \\
\hline 1 & ENSG00000267368 & UPK3BL1 & 5.136 & 35.159 & $2.52 \times 10^{-6}$ & Up \\
2 & ENSG00000197603 & CPLANE1 & -4.547 & 0.043 & $2.25 \times 10^{-5}$ & Down \\
3 & ENSG00000151967 & SCHIP1 & -4.411 & 0.047 & $4.55 \times 10^{-6}$ & Down \\
4 & ENSG00000153956 & CACNA2D1 & -4.206 & 0.054 & $3.40 \times 10^{-5}$ & Down \\
5 & ENSG00000158528 & PPP1R9A & -4.179 & 0.055 & $4.67 \times 10^{-5}$ & Down \\
6 & ENSG00000228526 & MIR34AHG & -4.131 & 0.057 & $5.00 \times 10^{-6}$ & Down \\
7 & ENSG00000133110 & POSTN & -3.563 & 0.085 & $4.15 \times 10^{-7}$ & Down \\
8 & ENSG00000214827 & MTCP1 & -3.425 & 0.093 & $2.55 \times 10^{-6}$ & Down \\
9 & ENSG00000210140 & MT-TC & 3.382 & 10.423 & $8.75 \times 10^{-8}$ & Down \\
10 & ENSG00000120262 & CCDC170 & -3.147 & 0.113 & $2.42 \times 10^{-5}$ & Down \\
11 & ENSG00000171466 & ZNF562 & -2.795 & 0.144 & $8.90 \times 10^{-9}$ & Down \\
12 & ENSG00000165392 & WRN & -2.636 & 0.161 & $1.35 \times 10^{-5}$ & Down \\
13 & ENSG00000142731 & PLK4 & -2.500 & 0.177 & $8.87 \times 10^{-5}$ & Down \\
14 & ENSG00000198689 & SLC9A6 & -2.459 & 0.182 & $6.73 \times 10^{-6}$ & Down \\
15 & ENSG00000113580 & NR3C1 & -2.419 & 0.187 & $2.23 \times 10^{-5}$ &
\end{tabular}

FC, fold change.
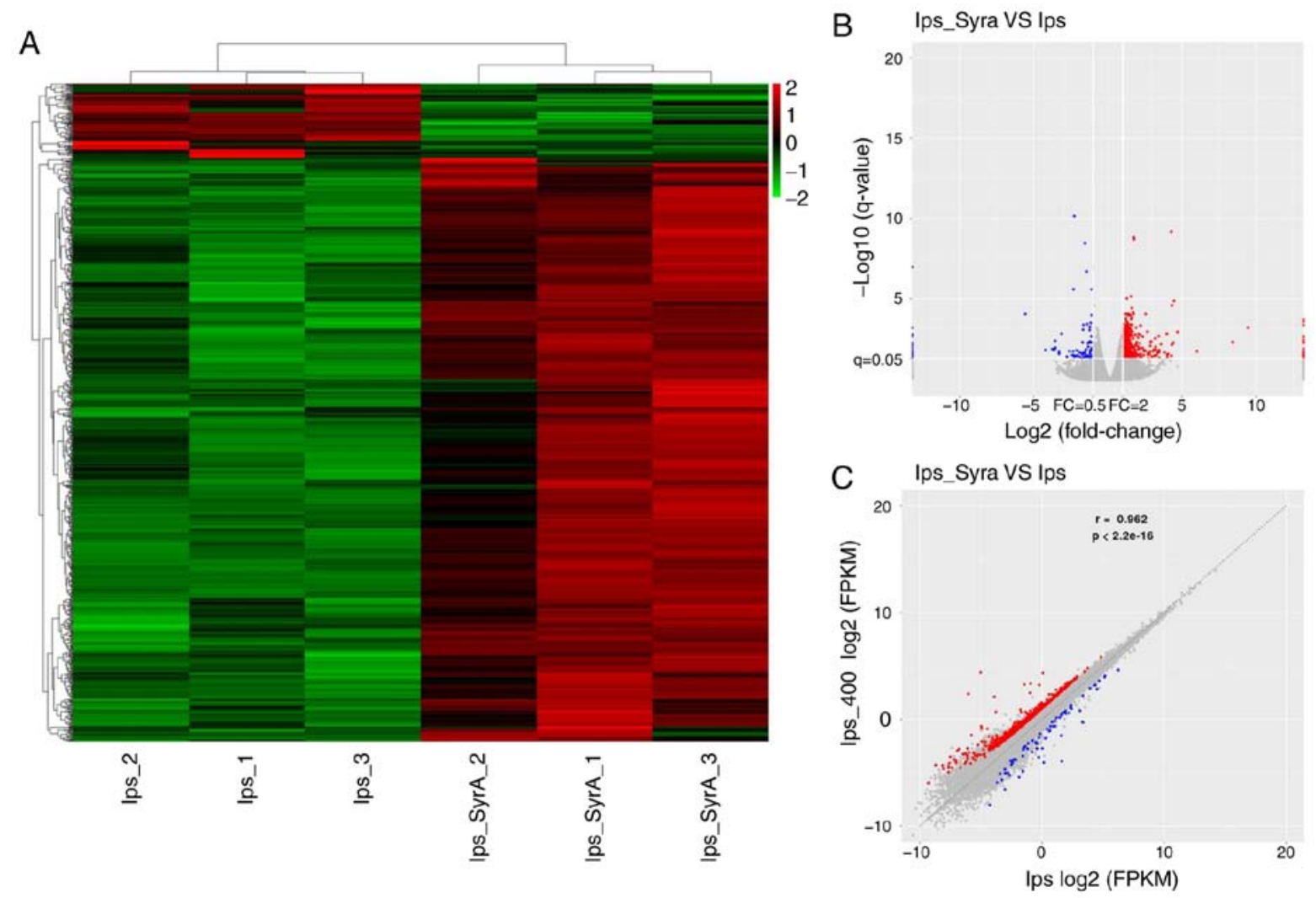

Figure 4. RNA-Seq analysis of LPS-stimulated NP cells treated with SyrA. (A) Heat map of DEGs from the SyrA-treatment group (SyrA plus LPS group vs. LPS group). (B) Volcano plots of 727 upregulated and 92 downregulated DEGs. The vertical lines represent $a b s\left(\log _{2} \mathrm{FC}\right)=1$, and the thick horizontal line indicates $\mathrm{P}=0.05$. (C) Scatter plots of the genes with abs $\left(\log _{2} \mathrm{FC}\right)>1$ in SyrA-reversed groups. Group 'lps_SyrA' indicates the SyrA plus LPS treatment group and 'lps' indicates the LPS treatment group. LPS, lipopolysaccharide; NP, nucleus pulposus; SyrA, syringic acid; DEGs, differentially expressed genes; abs, absolute; FC, fold change.

$R T-q P C R$ validation of the RNA-Seq analysis. The FC of the 65 DEGs from the LPS-stimulated groups was compared with that of the 819 DEGs from the SyrA-reversed groups to screen the genes that were regulated by both LPS and SyrA, which therefore could have an important role in preventing apoptosis. As a result, 33 DEGs were screened, i.e., nearly 
Table III. Top 15 differentially expressed genes from the syringic acid-reversed groups.

\begin{tabular}{rllrrrr}
\hline No. & \multicolumn{1}{c}{ Gene ID } & \multicolumn{1}{c}{ Gene name } & log2FC & FC & P-value & Up/downregulated \\
\hline 1 & ENSG00000267368 & UPK3BL1 & -5.558 & 0.021 & $8.55 \times 10^{-8}$ & Down \\
2 & ENSG00000114790 & ARHGEF26 & 4.423 & 21.446 & $6.89 \times 10^{-5}$ & Up \\
3 & ENSG00000231292 & IGKV1OR2-108 & -4.206 & 0.054 & $4.18 \times 10^{-4}$ & Down \\
4 & ENSG00000237248 & LINC00987 & 4.013 & 16.142 & $2.54 \times 10^{-3}$ & Up \\
5 & ENSG00000164669 & INTS4P1 & 3.989 & 15.877 & $7.96 \times 10^{-6}$ & Up \\
6 & ENSG00000197978 & GOLGA6L9 & 3.822 & 14.147 & $7.75 \times 10^{-4}$ & Up \\
7 & ENSG00000086696 & HSD17B2 & -3.739 & 0.075 & $3.34 \times 10^{-4}$ & Down \\
8 & ENSG00000284118 & MIR4707 & -3.653 & 0.079 & $4.09 \times 10^{-4}$ & Down \\
9 & ENSG00000237380 & HOXD-AS2 & -3.557 & 0.085 & $3.13 \times 10^{-04}$ & Down \\
10 & ENSG00000005471 & ABCB4 & 3.476 & 11.123 & $3.02 \times 10^{-3}$ & Up \\
11 & ENSG00000153956 & CACNA2D1 & 3.468 & 11.069 & $4.39 \times 10^{-4}$ & Up \\
12 & ENSG00000187790 & FANCM & 3.443 & 10.874 & $4.97 \times 10^{-4}$ & Up \\
13 & ENSG00000283824 & MIR22 & 3.435 & 10.817 & $1.41 \times 10^{-5}$ & Up \\
14 & ENSG00000021645 & NRXN3 & 3.379 & 10.400 & $8.66 \times 10^{-4}$ & Up \\
15 & ENSG00000198088 & NUP62CL & 3.356 & 10.239 & $7.47 \times 10^{-4}$ & \\
\hline
\end{tabular}

FC, fold change.

half of the total DEGs of the LPS-stimulated group (Table SI). Among the top 15 most significantly dysregulated DEGs, the expression of seven was completely reversed following SyrA treatment (Table IV), and RT-qPCR analysis indicated that the expression of UPK3BL1 was significantly upregulated after LPS stimulation, and subsequently downregulated by SyrA (Fig. 6). In addition, the altered expression patterns of CACNA2D1 and polo-like kinase 4 (PLK4) were consistent with the RNA-Seq results (Fig. 6).

Bioinformatics analyses. For an improved understanding of these DEGs, GO analysis was conducted. As shown in Fig. 7A and B, there was a high correspondence of DEGs from LPS-stimulated groups and SyrA-reversed groups in terms of the GO classifications. The 65 DEGs were mostly annotated for 'biological regulation', 'cellular process' and 'single-organism process' within the 'Biological Process'; 'cell', 'cell part' and 'organelle' within 'Cellular Component'; and 'binding' followed by 'catalytic activity' within 'Molecular Function' (Fig. 7A). Similar distribution trends could also be found among the 819 DEGs in the SyrA-reversed groups (Fig. 7B). KEGG classification analysis was also conducted: The top 30 pathways were screened, and are presented in Fig. 8. As shown in Fig. 8A, DEGs from LPS-stimulated groups were mainly enriched in 'Cardiac muscle contraction', 'Endocytosis' and the 'MAPK signaling pathway'. In the SyrA-reversed group, DEGs were involved in the 'Regulation of the actin cytoskeleton', 'Ubiquitin-mediated proteolysis', 'ErbB signaling pathway', 'Autophagy-other', 'Cellular senescence' and 'Apoptosis-multiple species' (Fig. 8B).

\section{Discussion}

To date, studies have primarily focused on the mechanisms of apoptosis of NP cells based on RNAs and long non-coding RNAs (17-19). However, significant obstacles still remain in terms of their clinical application, such as poor uptake, low potency at target sites, and off-target effects (20). Consequently, seeking novel molecules to prevent the apoptosis of NP cells and further slow IDD might be a more feasible option for clinical implementation. The present study identified that SyrA was able to reverse both apoptosis and the abnormal expression of certain mRNAs in the NP cells that were induced by LPS.

LPS is a stimulant that has been used to induce cellular apoptosis, including apoptosis of NP cells $(12,13,21)$. The stimulatory action of LPS on NP cells not only involves the initiation of apoptosis, but also promotes the expression of ECM-degrading enzymes, thereby accelerating IDD $(22,23)$. However, the experimental concentration of LPS that was used to induce NP cellular apoptosis in these studies varied widely, ranging between $10 \mathrm{ng} / \mathrm{ml}$ and $10 \mu \mathrm{g} / \mathrm{ml}(12,13)$. Therefore, in the present study, different concentrations of LPS were used with NP cells in culture, and the results showed that $0.01 \mu \mathrm{g} / \mathrm{ml}(10 \mathrm{ng} / \mathrm{ml})$ LPS induced apoptosis the most efficiently, consistent with the results of Liu et al (13). Notably, the LPS concentration and its apoptosis induction rate were found to be negatively related, in contrast with other studies $(12,24)$. A previous study showed that no significant survival inhibition was observed after nasal epithelial cells were treated with a high dose of LPS, which was demonstrated to be the autophagy activator (25). Hence, it was hypothesized that the negative relationship between LPS concentration and survival inhibition could have been caused by autophagy initiated by a higher dose of LPS.

Another study, NP cells pretreated with SyrA then treated with LPS, was also conducted and the results indicated that SyrA could exert a protection effect compared to cells treated with LPS alone, which is similar to the results in the present study (cells treated with SryA and LPS together). Then, the easier treatment way, treated with SryA and LPS together, was selected for further study. 

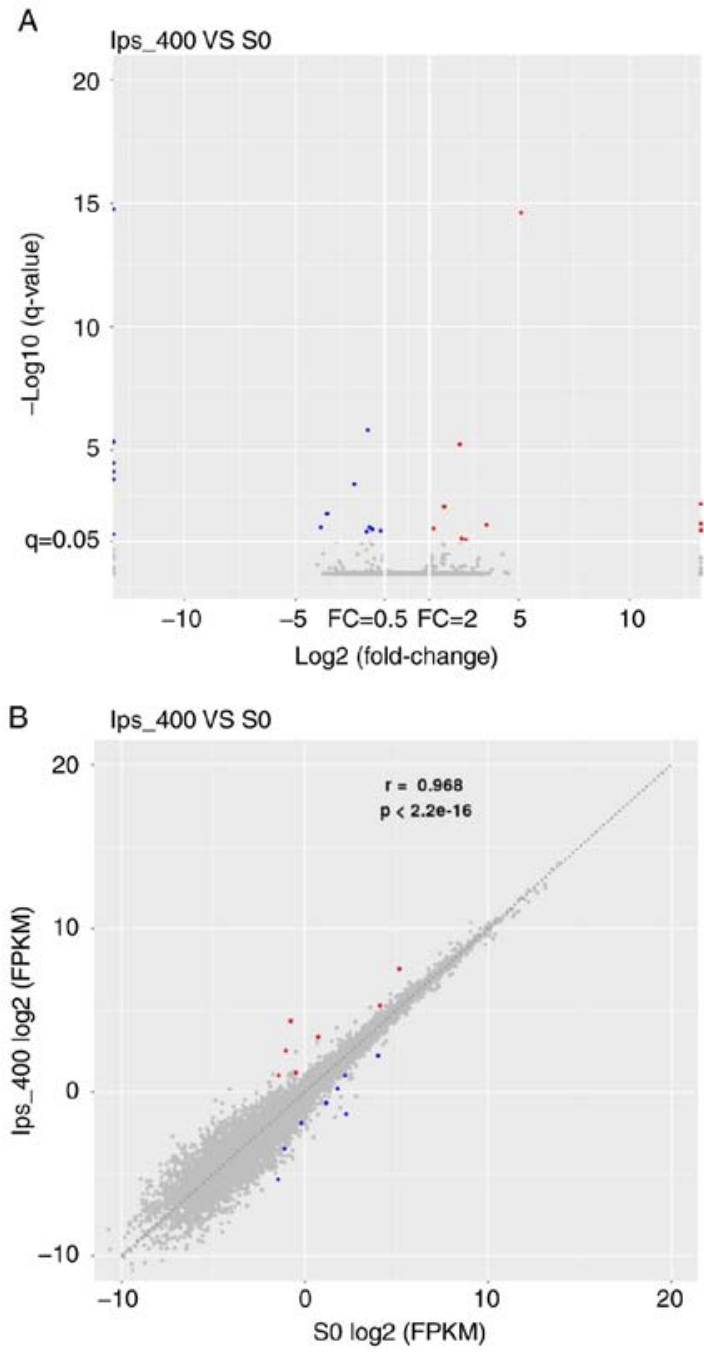

Figure 5. RNA-Seq analysis of DEGs from the SyrA plus LPS treatment group and control group. (A) Volcano plots of 10 upregulated and 15 downregulated DEGs. The vertical lines represent abs $\left(\log _{2} \mathrm{FC}\right)=1$, and the thick horizontal line indicates $P=0.05$. (B) Scatter plots of genes with $\operatorname{abs}\left(\log _{2} \mathrm{FC}\right)>1$. Group 'lps_SyrA' indicates the SyrA plus LPS treatment group and 'S0' indicates the control group. DEGs, differentially expressed genes; SyrA, syringic acid; LPS, lipopolysaccharide; abs, absolute; FC, fold change.

Usually, RNA-Seq is used to analyze the DEGs between two groups; in the present study, the mRNA expression in three groups were detected, and DEGs were identified between the pairing of groups. The major objective of the present study was to search for the main target genes that are involved in both LPS stimulation and SyrA reversion, even though the total effect of SyrA interference on LPS-stimulated NP cells compared with the control NP cells was also observed. In the LPS-stimulated groups, a total of 65 DEGs were identified from 819 DEGs following treatment with SyrA. The effect of SyrA on LPS-stimulated NP cells was greater compared with that of LPS on NP cells. This suggested that there would be a greater influence of SyrA plus LPS on the transcriptome of NP cells. In contrast to this, the results demonstrated that the alterations in the expression of the 65 DEGs were reversed by treatment with SyrA, which made the abnormally expressed transcriptome of LPS-stimulated NP cells return to a relatively normal phenotype.

The comparison between the top 15 DEGs from the LPS-stimulated groups and the 819 DEGs from the

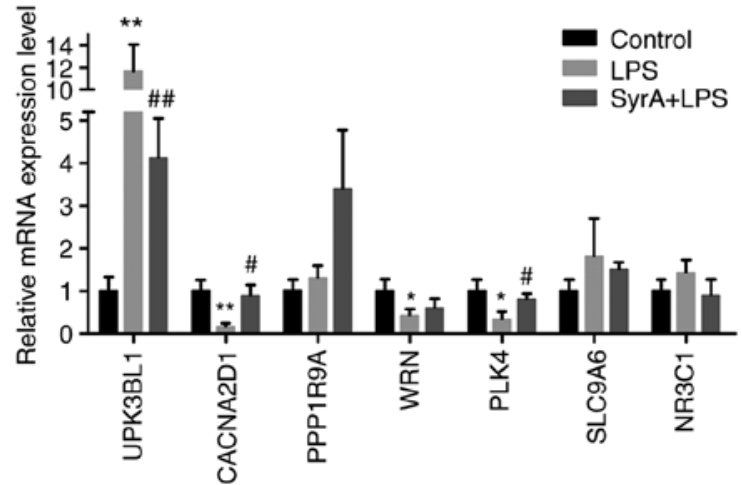

Figure 6. Reverse transcription-quantitative PCR validation of seven differentially expressed genes from RNA-Seq analyses ${ }^{*} \mathrm{P}<0.05,{ }^{* *} \mathrm{P}<0.01$ vs. Control; ${ }^{\#} \mathrm{P}<0.05,{ }^{\# \#} \mathrm{P}<0.01$ vs. LPS. LPS, lipopolysaccharide; SyrA, syringic acid.

SyrA-reversed groups showed that seven were involved in LPS stimulation and SyrA reversion. The results of RT-qPCR validation screened three DEGs (CACNA2D1, PLK4 and UPK3BL1), which were in line with the trend of each FC. CACNA2D1 is a subunit of the $\mathrm{Ca}^{2+}$-channel complex, and to the best of the authors' knowledge, no research has previously reported the role of this gene in apoptosis. However, the protein CACNA2D1 shares a similar secondary and tertiary structure with CACNA2D2, which is reported to be able to directly regulate the apoptosis of non-small cell lung cancer cells, and its regulation was associated with disruption of the mitochondrial membrane integrity (26). These data suggest that there might be some as yet unknown relationship between CACNA2D1 and LPS-induced apoptosis of NP cells. Studies involved in the regulation of apoptosis by PLK4, a unique member of the PLK family, are relatively abundant. For instance, the depletion of PLK4 significantly promoted apoptosis of hepatocellular carcinoma cells and suppressed cell proliferation and tissue invasion (27). In neuroblastoma cells, downregulation of PLK4 could also dramatically increase the rate of apoptosis and lead to an inhibition of cell migratory and invasive abilities (28). Additionally, apoptotic death occurred in lung cancer cells after treatment with CFI-400945, a PLK4 inhibitor (29). In the present study, the expression of PLK4 was downregulated in LPS-induced apoptosis and reversed by SyrA treatment. The relationship between the expression level of PLK4 and apoptosis inducement/inhibition in NP cells identified in the present study was consistent with the aforementioned three studies. This indicated that PLK4 possibly exerts important roles in both LPS-induced and SyrA-reversed apoptosis of the NP cells. Until now, to the best of the authors' knowledge, no studies have been published on the role of UPK3BL1: Its potentially dominant effect on the apoptosis of NP cells is an hypothesis that requires further experimental validation.

Compared with GO classification analysis, KEGG pathway enrichment analysis appeared to have a higher level of precision and helped the study in enabling us to look for some potential genes involved in the apoptosis of NP cells, as well as other mechanisms responsible for survival induced by SyrA. The KEGG pathway analysis revealed that DEGs from LPS-stimulated groups were enriched in the MAPK signaling 
Table IV. The FC of seven DEGs out of the top 15 DEGs in LPS-stimulated groups were reversed after SyrA treatment.

\begin{tabular}{|c|c|c|c|c|c|c|c|}
\hline \multirow[b]{2}{*}{ No. } & \multirow[b]{2}{*}{ Gene name } & \multicolumn{3}{|c|}{ LPS-stimulated groups } & \multicolumn{3}{|c|}{ SyrA-reversed groups } \\
\hline & & $\log 2 \mathrm{FC}$ & $\mathrm{FC}$ & Up/downregulated & $\log 2 \mathrm{FC}$ & $\mathrm{FC}$ & Up/downregulated \\
\hline 1 & UPK3BL1 & 5.136 & 35.159 & Up & -5.558 & 0.021 & Down \\
\hline 2 & CACNA2D1 & -4.206 & 0.054 & Down & 3.468 & 11.069 & Up \\
\hline 3 & PPP1R9A & -4.179 & 0.055 & Down & 3.279 & 9.707 & Up \\
\hline 4 & WRN & -2.636 & 0.161 & Down & 2.207 & 4.618 & Up \\
\hline 5 & PLK4 & -2.500 & 0.177 & Down & 1.578 & 2.985 & Up \\
\hline 6 & SLC9A6 & -2.459 & 0.182 & Down & 1.751 & 3.367 & Up \\
\hline 7 & NR3C1 & -2.419 & 0.187 & Down & 1.711 & 3.275 & Up \\
\hline
\end{tabular}

DEG, differentially expressed gene; LPS, lipopolysaccharide; SyrA, syringic acid; FC, fold change.

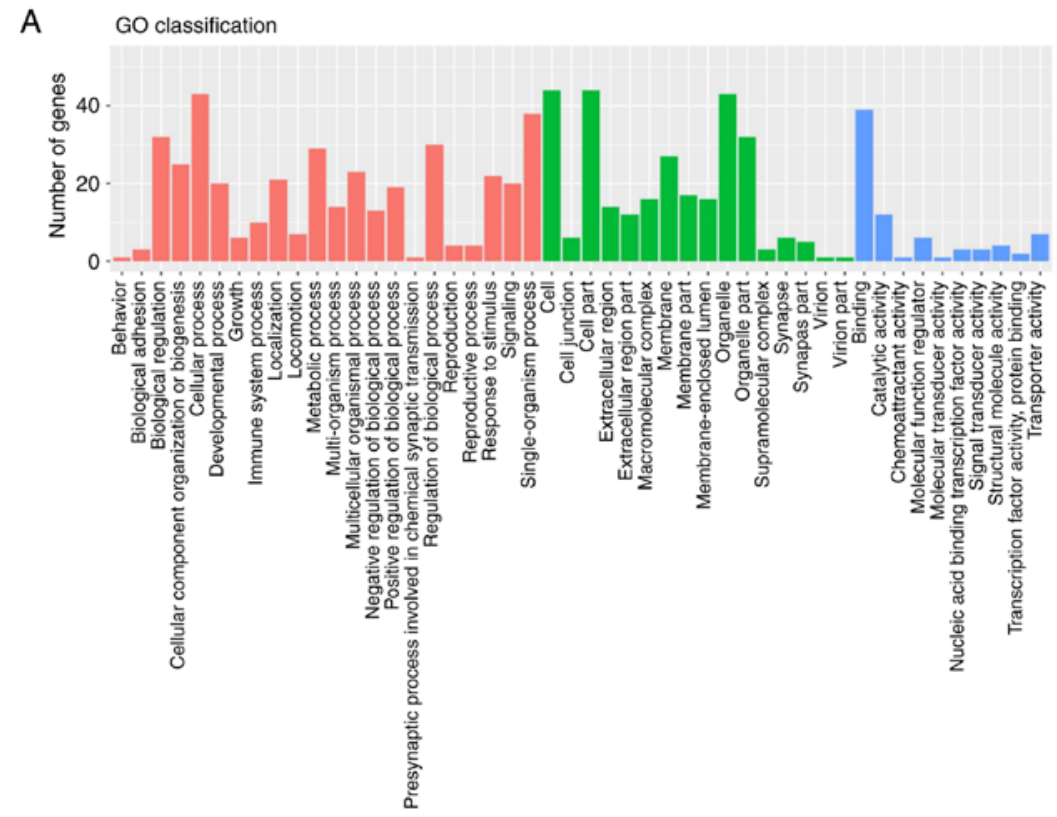

GO_domain

Biological_process

Molecular_function

B

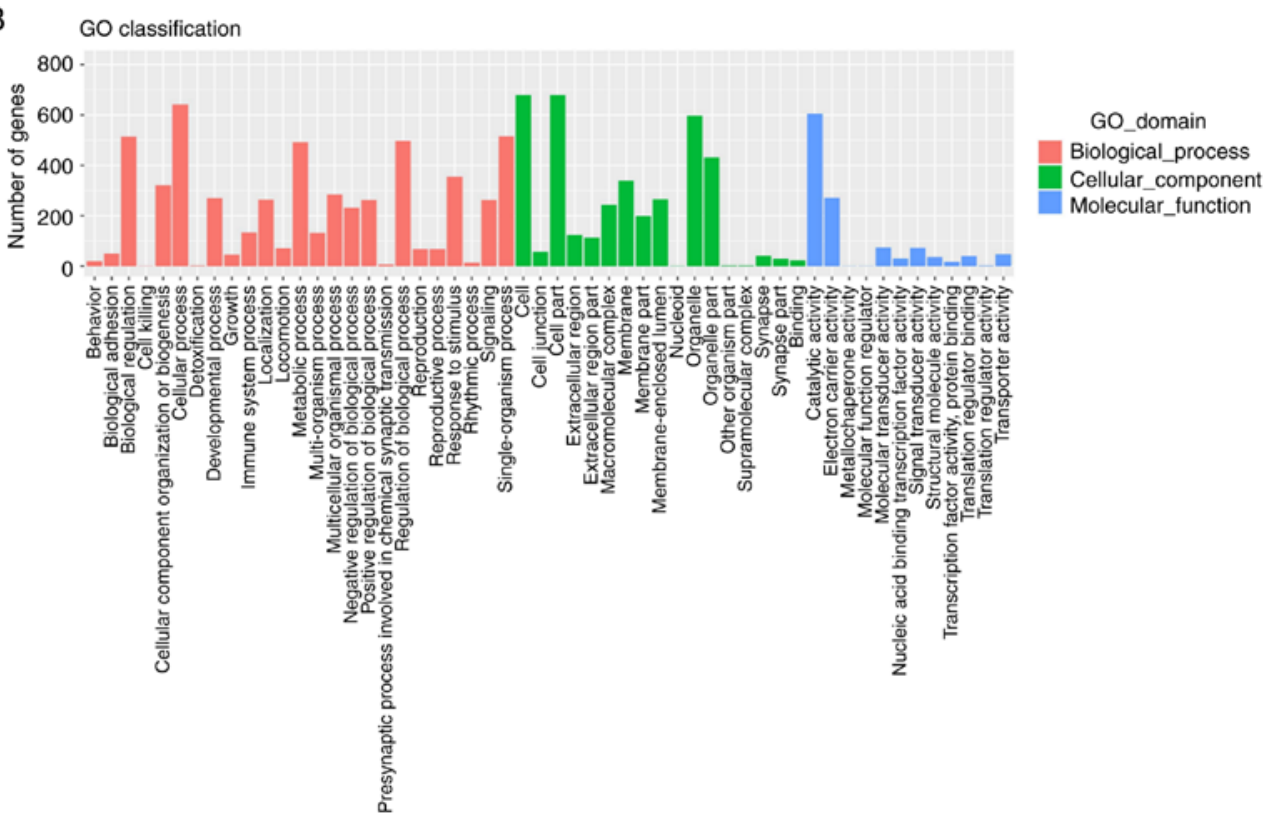

Figure 7. GO analyses. (A) GO analysis of DEGs from the LPS-stimulated groups. (B) GO analysis of DEGs from the syringic acid plus LPS-treated groups. GO, Gene Ontology; DEG, differentially expressed genes; LPS, lipopolysaccharide. 
A

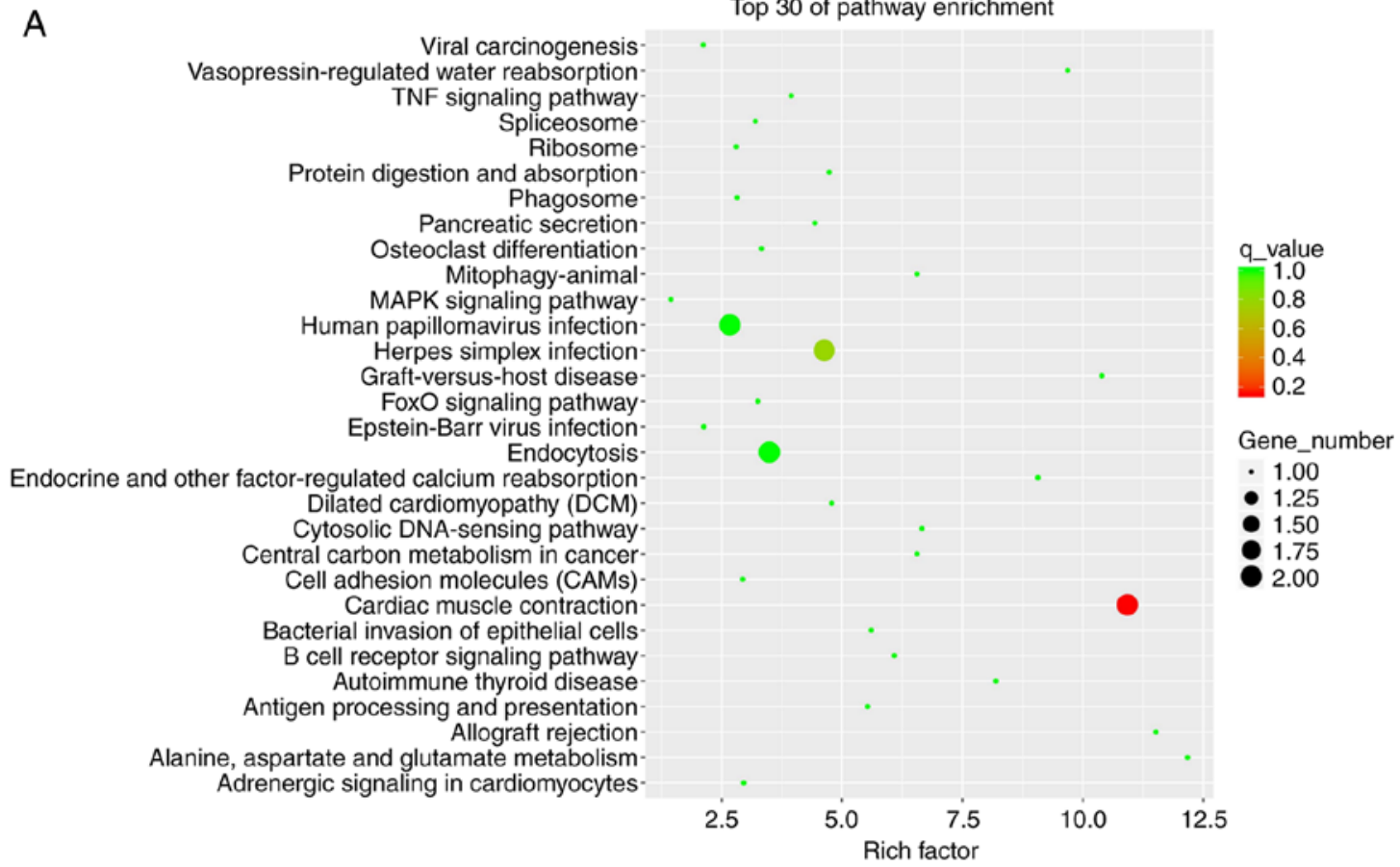

B

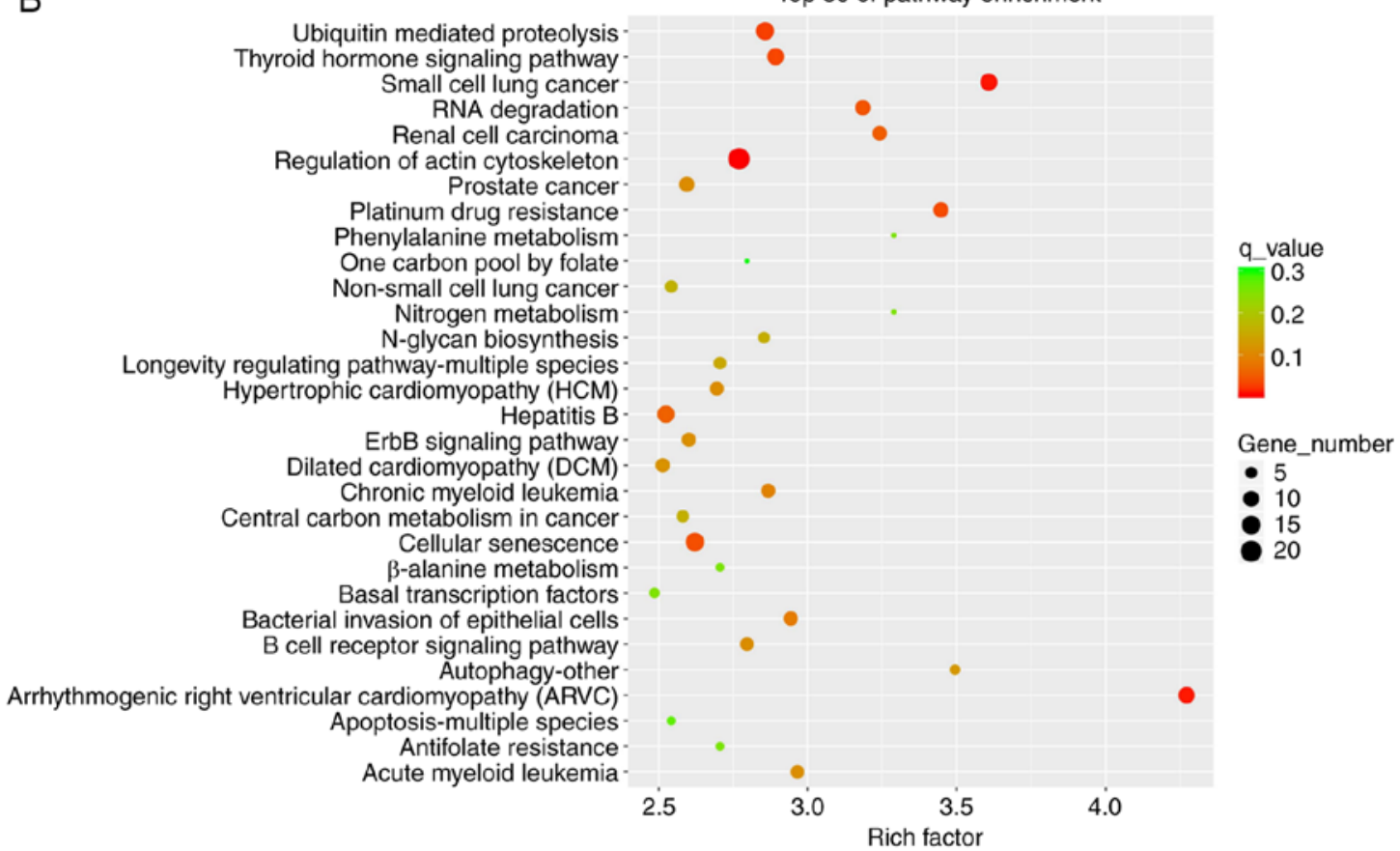

Figure 8. KEGG analyses. (A) KEGG analysis of DEGs from LPS-stimulated groups. (B) KEGG analysis of DEGs from the syringic acid plus LPS-treated groups. KEGG, Kyoto Encyclopedia of Genes and Genomes; DEGs, differentially expressed genes; LPS, lipopolysaccharide.

pathway. The role of the MAPK pathway in the apoptosis of NP cells has previously been reported $(30,31)$, and the MAPK pathway might be involved in the apoptosis observed in the present study. A DEG identified that is known to be involved with the MAPK pathway was CACNA2D1, which could also indicate the potential role of CACNA2D1 in the apoptosis of NP cells.

Following treatment with SyrA, DEGs were found to be enriched in pathways of interest to the present study, such as the 'Autophagy-other' and 'Apoptosis-multiple species' pathways. The number of DEGs in the 'Autophagy-other' pathway was 14 (consisting of BIRC3, CUL5, CUL2, HERC3, WWP1, NEDD4, UBR5, MID1, UBA6, ITCH, UBE4A, UBE2N, CUL4B and HERC1). Autophagy is known to be an effective and important way for cells to survive in an unfavorable environment (32). The viability of the damaged NP cells reversed by SyrA was not limited to the inhibition of apoptosis, and autophagy might also be initiated after SyrA treatment. Among the 14 DEGs, the majority have important roles in autophagy (33-35); however, their effects on the autophagy of NP cells are still unknown and require further research. Notably, the level of cullin 4B (CUL4B) was found to be 
significantly upregulated in IDD samples compared with the controls, and this was positively correlated with the severity of IDD (36). In addition, the expression of CUL4B was enhanced after treatment with the inflammatory factors interleukin (IL)-6 and tumour necrosis factor (TNF)- $\alpha$, and reversed by IL-6 and TNF- $\alpha$ inhibitors in primary NP cells (37). The previously reported close association between CUL4B and inflammation suggested that there might an anti-inflammatory effect exerted by SyrA in the survival of damaged NP cells. Indeed, the onset of inflammation induced by LPS in NP cells and the anti-inflammatory effect of SyrA has been confirmed in many prior reports (37-41). DEGs in the 'Apoptosis-multiple species' pathway include APAF1, BIRC3 and BCL2. The role of $\mathrm{Bcl}-2$ in apoptotic NP cells has been demonstrated in previous studies $(18,41)$, including in the work presented here. The other two DEGs might also have a role in the apoptosis of NP cells, but further studies are still needed to confirm the roles of these proteins.

Collectively, the present study has shown that SyrA can reverse the LPS-induced apoptosis of NP cells, which was attributed to an extensive reversion of the dysregulated mRNA levels to near-normal expression levels. The regulation of the CACNA2D1 and PLK4 genes may be responsible for the reversal of apoptosis. Additionally, the complete survival reversion induced by SyrA on damaged NP cells might be due to the onset of autophagy, which prevents apoptosis and promotes cell viability. All these hypotheses require further study to help elucidate the potential mechanisms for apoptosis/survival reversion induced by SyrA in NP cells.

\section{Acknowledgements}

Not applicable.

\section{Funding}

This work was supported by Research Project of Inheritance and Innovation of Traditional Chinese Medicine and Ethnic Medicine in Health Department of Guangxi Zhuang Autonomous Region (grant no. GZPT13-41).

\section{Availability of data and materials}

The datasets used and/or analyzed during the current study are available from the corresponding author on reasonable request.

\section{Authors' contributions}

$\mathrm{CZ}$ and QF designed and supervised the project. HZ and HQ performed the major research and wrote the manuscript. YY, JS and YT analyzed and interpreted the data. All authors read and approved the final manuscript.

\section{Ethics approval and consent to participate}

Not applicable.

\section{Patient consent for publication}

Not applicable.

\section{Competing interests}

The authors declare that they have no competing interests.

\section{References}

1. GBD 2017 Disease and Injury Incidence and Prevalence Collaborators: Global, regional, and national incidence, prevalence, and years lived with disability for 354 diseases and injuries for 195 countries and territories, 1990-2017: A systematic analysis for the Global Burden of Disease Study 2017. Lancet 392: 1789-1858, 2018.

2. Vadala G, Russo F, Di Martino A and Denaro V: Intervertebral disc regeneration: From the degenerative cascade to molecular therapy and tissue engineering. J Tissue Eng Regen Med 9: 679-690, 2015.

3. Sakai D and Grad S: Advancing the cellular and molecular therapy for intervertebral disc disease. Adv Drug Deliv Rev 84: 159-171, 2015.

4. Sampara P, Banala RR, Vemuri SK, Av GR and Gpv S: Understanding the molecular biology of intervertebral disc degeneration and potential gene therapy strategies for regeneration: A review. Gene Ther 25: 67-82, 2018.

5. Ding F, Shao ZW and Xiong LM: Cell death in intervertebral disc degeneration. Apoptosis 18: 777-785, 2013.

6. Srinivasulu C, Ramgopal M, Ramanjaneyulu G, Anuradha CM and Suresh Kumar C: Syringic acid (SA) a review of its occurrence, biosynthesis, pharmacological and industrial importance. Biomed Pharmacother 108: 547-557, 2018.

7. Cao Y, Zhang L, Sun S, Yi Z, Jiang X and Jia D: Neuroprotective effects of syringic acid against OGD/R-induced injury in cultured hippocampal neuronal cells. Int J Mol Med 38: 567-573, 2016.

8. Song M, Du Z, Lu G, Li P and Wang L: Syringic acid protects retinal ganglion cells against $\mathrm{H}_{2} \mathrm{O}_{2}$-induced apoptosis through the activation of PI3K/Akt signaling pathway. Cell Mol Biol (Noisy-le-grand) 62: 50-54, 2016.

9. Ding SK, Wang LX, Guo LS, Luo P, Du JJ, Zhao ZL and Wang GG: Syringic acid inhibits apoptosis pathways via downregulation of p38MAPK and JNK signaling pathways in $\mathrm{H} 9 \mathrm{c} 2$ cardiomyocytes following hypoxia/reoxygenation injury. Mol Med Rep 16: 2290-2294, 2017.

10. Sancak EB, Akbas A, Silan C, Cakir DU, Turkon H and Ozkanli SS: Protective effect of syringic acid on kidney ischemia-reperfusion injury. Ren Fail 38: 629-635, 2016.

11. Wang S, Sun J, Yang H, Zou W, Zheng B, Chen Y, Guo Y and Shi J: Profiling and bioinformatics analysis of differentially expressed circular RNAs in human intervertebral disc degeneration. Acta Biochim Biophys Sin (Shanghai) 51: 571-579, 2019

12. Chai X, Si H, Song J, Chong Y, Wang J and Zhao G: miR-486-5p inhibits inflammatory response, matrix degradation and apoptosis of nucleus pulposus Cells through directly targeting FOXO1 in intervertebral disc degeneration. Cell Physiol Biochem 52: 109-118, 2019.

13. Liu J, Jiang T, He M, Fang D, Shen C, Le Y, He M, Zhao J and Zheng L: Andrographolide prevents human nucleus pulposus cells against degeneration by inhibiting the NF-kappaB pathway. J Cell Physiol 234: 9631-9639, 2019.

14. Livak KJ and Schmittgen TD: Analysis of relative gene expression data using real-time quantitative PCR and the 2(-Delta Delta C(T)) method. Methods 25: 402-408, 2001.

15. The Gene Ontology Consortium: The Gene Ontology Resource: 20 years and still Going strong. Nucleic Acids Res 47: D330-D338, 2019.

16. Kanehisa M, Sato Y, Furumichi M, Morishima K and Tanabe M: New approach for understanding genome variations in KEGG. Nucleic Acids Res 47: D590-D595, 2019.

17. Yang Q, Guo XP, Cheng YL and Wang Y: MicroRNA-143-5p targeting eEF2 gene mediates intervertebral disc degeneration through the AMPK signaling pathway. Arthritis Res Ther 21: 97, 2019.

18. Wang R, Wen B and Sun D: miR-573 regulates cell proliferation and apoptosis by targeting Bax in nucleus pulposus cells. Cell Mol Biol Lett 24: 2, 2019.

19. Yu Y, Zhang X, Li Z, Kong L and Huang Y: LncRNA HOTAIR suppresses TNF-alpha induced apoptosis of nucleus pulposus cells by regulating miR-34a/Bcl-2 axis. Biomed Pharmacother 107: 729-737, 2018. 
20. Barata P, Sood AK and Hong DS: RNA-targeted therapeutics in cancer clinical trials: Current status and future directions. Cancer Treat Rev 50: 35-47, 2016.

21. Zhang Y, Yang J, Zhou X, Wang N, Li Z, Zhou Y, Feng J, Shen D and Zhao W: Knockdown of miR-222 inhibits inflammation and the apoptosis of LPS-stimulated human intervertebral disc nucleus pulposus cells. Int J Mol Med 44: 1357-1365, 2019.

22. Liu H, Pan H, Yang H, Wang J, Zhang K, Li X, Wang H, Ding W, Li $\mathrm{B}$ and Zheng Z: LIM mineralization protein-1 suppresses TNF-alpha induced intervertebral disc degeneration by maintaining nucleus pulposus extracellular matrix production and inhibiting matrix metalloproteinases expression. J Orthop Res 33: 294-303, 2015.

23. Zhongyi S, Sai Z, Chao L and Jiwei T: Effects of nuclear factor kappa B signaling pathway in human intervertebral disc degeneration. Spine (Phila Pa 1976) 40: 224-232, 2015.

24. Li B, Zhang H, Zeng M, He W, Li M, Huang X, Deng DY and $\mathrm{Wu} \mathrm{J}$ : Bone marrow mesenchymal stem cells protect alveolar macrophages from lipopolysaccharide-induced apoptosis partially by inhibiting the Wnt/ $\beta$-catenin pathway. Cell Biol Int 39: 192-200, 2015

25. Wang XH, Zhang ZH, Cai XL, Ye P, Feng X, Liu TT and Li XZ: Lipopolysaccharide induces autophagy by targeting the AMPK-mTOR pathway in human nasal epithelial cells. Biomed Pharmacother 96: 899-904, 2017.

26. Carboni GL, Gao B, Nishizaki M, Xu K, Minna JD, Roth JA and Ji L: CACNA2D2-mediated apoptosis in NSCLC cells is associated with alterations of the intracellular calcium signaling and disruption of mitochondria membrane integrity. Oncogene 22: 615-626, 2003.

27. Bao J, Yu Y, Chen J, He Y, Chen X, Ren Z, Xue C, Liu L, Hu Q, $\mathrm{Li}$ J, et al: MiR-126 negatively regulates PLK-4 to impact the development of hepatocellular carcinoma via ATR/CHEK1 pathway. Cell Death Dis 9: 1045, 2018.

28. Tian X, Zhou D, Chen L, Tian Y, Zhong B, Cao Y, Dong Q, Zhou M, Yan J, Wang Y, et al: Polo-like kinase 4 mediates epithelial-mesenchymal transition in neuroblastoma via PI3K/Akt signaling pathway. Cell Death Dis 9: 54, 2018.

29. Kawakami M, Mustachio LM, Zheng L, Chen Y, RodriguezCanales J, Mino B, Kurie JM, Roszik J, Villalobos PA, Thu KL, et al: Polo-like kinase 4 inhibition produces polyploidy and apoptotic death of lung cancers. Proc Natl Acad Sci USA 115: 1913-1918, 2018

30. Wang T, Wang CJ, Tian S and Song HB: Overexpressed IGFBP5 promotes cell proliferation and inhibits apoptosis of nucleus pulposus derived from rats with disc degeneration through inactivating the ERK/MAPK axis. J Cell Biochem 120: 18782-18792, 2019.
31. Xu Q, Fang H, Zhao L, Zhang C, Zhang L and Tian B: Mechano growth factor attenuates mechanical overload-induced nucleus pulposus cell apoptosis through inhibiting the p38 MAPK pathway. Biosci Rep 39: BSR20182462, 2019.

32. Jiang L, Yuan F, Yin X and Dong J: Responses and adaptations of intervertebral disc cells to microenvironmental stress: A possible central role of autophagy in the adaptive mechanism. Connect Tissue Res 55: 311-321, 2014

33. Antonioli M, Albiero F, Nazio F, Vescovo T, Perdomo AB, Corazzari M, Marsella C, Piselli P, Gretzmeier C, Dengjel J, et al: AMBRA1 interplay with cullin E3 ubiquitin ligases regulates autophagy dynamics. Dev Cell 31: 734-746, 2014.

34. Xie W, Jin S and Cui J: The NEDD4-USP13 axis facilitates autophagy via deubiquitinating PIK3C3. Autophagy 16: 1150-1151, 2020

35. Rossi M, Rotblat B, Ansell K, Amelio I, Caraglia M, Misso G, Bernassola F, Cavasotto CN, Knight RA, Ciechanover A and Melino G: High throughput screening for inhibitors of the HECT ubiquitin E3 ligase ITCH identifies antidepressant drugs as regulators of autophagy. Cell Death Dis 5: e1203, 2014.

36. Chen Z, Han Y, Deng C, Chen W, Jin L, Chen H, Wang K, Shen $\mathrm{H}$ and Qian L: Inflammation-dependent downregulation of miR-194-5p contributes to human intervertebral disc degeneration by targeting CUL4A and CUL4B. J Cell Physiol 234: 19977-19989, 2019

37. Wang $\mathrm{H}$, Hao P, Zhang $\mathrm{H}$, Xu C and Zhao J: MicroRNA-223 inhibits lipopolysaccharide-induced inflammatory response by directly targeting Irak1 in the nucleus pulposus cells of intervertebral disc. IUBMB Life 70: 479-490, 2018.

38. Guo F, Zou Y and Zheng Y: Moracin M inhibits lipopolysaccharide-induced inflammatory responses in nucleus pulposus cells via regulating PI3K/Akt/mTOR phosphorylation. Int Immunopharmacol 58: 80-86, 2018.

39. Ham JR, Lee HI, Choi RY, Sim MO, Seo KI and Lee MK: Anti-steatotic and anti-inflammatory roles of syringic acid in high-fat diet-induced obese mice. Food Funct 7: 689-697, 2016.

40. Li Y, Zhang L, Wang X, Wu W and Qin R: Effect of Syringic acid on antioxidant biomarkers and associated inflammatory markers in mice model of asthma. Drug Dev Res 80: 253-261, 2019.

41. Zhu H, Sun B and Shen Q: TNF- $\alpha$ induces apoptosis of human nucleus pulposus cells via activating the TRIM14/NF- $\kappa \mathrm{B}$ signalling pathway. Artif Cells Nanomed Biotechnol 47: 3004-3012, 2019. International (CC BY-NC-ND 4.0) License. 